\title{
Visco-Elastic MHD Free Convective Flow through Porous Media in Presence of Radiation and Chemical Reaction with Heat and Mass Transfer
}

\author{
R. Choudhury ${ }^{1}$ and S. Kumar Das ${ }^{2 \dagger}$ \\ ${ }^{1}$ Department of Mathematics, Gauhati University, Guwahati-781 014, Assam, India \\ ${ }^{2}$ Department of Mathematics, Bajali College, Pathsala, Barpeta, Assam, India.
}

†Corresponding Author Email:sajall2003@yahoo.co.in

(Received April 3, 2013; accepted June 9, 2013)

\begin{abstract}
An analysis of visco-elastic free convective transient MHD flow over a vertical porous plate through porous media in presence of radiation and chemical reaction with heat and mass transfer is presented. A transverse variable suction velocity is applied on the porous plate. The equations governing the fluid flow, heat and mass transfer are solved by applying multiple perturbation technique. The expressions for transient velocity, temperature, species concentration and non-dimensional skin friction at the plate are obtained and the expressions for transient velocity and non-dimensional skin friction at the plate are illustrated graphically to observe the visco-elastic effect in combination of other flow parameters involved in the solution.
\end{abstract}

Keywords: MHD, Transient velocity, Chemical reaction, Radiation, Rarefaction parameter, Visco-elastic, skin friction, Grashof Number, Prandtl Number.

\section{NOMENCLATURE}

$A$ suction parameter.

$B_{0} \quad$ uniform transverse magnetic field.

$\bar{C}$ species concentration.

$C_{p} \quad$ specific heat at constant pressure.

$\bar{C}_{w}$ concentration at the plate surface.

$\overline{\mathrm{C}}_{\infty}$ concentration in fluid far away from plate.

$D$ chemical molecular diffusivity.

$G_{m}$ Grashof number for mass transfer.

$G_{r}$ Grashof number for heat transfer.

$h$ rarefaction parameter.

$K$ porosity parameter.

$K_{1} \quad$ visco-elastic parameter.

$K_{r}$ chemical reaction parameter.

$M$ magnetic parameter.

$P_{r} \quad$ Prandtl number.

$R$ radiation parameter.

$S_{c} \quad$ Schmidt number.

$\bar{T}$ fluid temperature.

$\bar{T}_{\infty}$ temperature in the free stream.

\section{INTRODUCTION}

The study of visco-elastic fluid flows over vertical surfaces immersed in porous media in presence of magnetic field has attracted the researchers because of its application in geophysics, astrophysics, geohydrology, chemical engineering, biological
$\bar{T}_{w} \quad$ temperature of the plate.

$u, v$ velocity components along $\mathrm{x}$-and $\mathrm{y}$ directions respectively.

$x, y$ Cartesian coordinates.

Greek Letters

$\beta \quad$ volumetric co-efficient of expansion for heat transfer.

$\bar{\beta}_{c} \quad$ volumetric co-efficient of expansion with species concentration.

$\varepsilon \quad$ perturbation parameter.

$\eta_{0} \quad$ limiting viscosity

$\Theta$ dimensionless temperature.

$K$ thermal conductivity of fluid.

$k_{e}$ mean absorption coefficient.

$P$ density of fluid.

$v$ kinematic viscosity.

$v_{0}$ mean suction velocity.

$\sigma_{s} \quad$ Stefan-Boltzmann constant.

$\varphi$ dimensionless concentration.

system, soil physics and filtration of solids from liquids. Also the studies of fluid flows through porous medium have become inevitable in the extraction of crude oil from the pores of rocks. The analysis of heat and mass transfer has attracted many researchers due to its application in geothermal and oil reservoir engineering studies. Sharma (2005) has studied the effect of fluctuating 
thermal and mass diffusion on unsteady free convective flow past a vertical plate in slip flow regime. Singh et al. (2010a) have investigated heat transfer over stretching surface in porous media in presence of magnetic field. Singh et al. (2010b) have studied MHD oblique stagnation point flow towards a stretching sheet with heat transfer. Singh et al. (2010c) have studied the effect of thermal radiation and magnetic field on unsteady stretching permeable sheet in presence of free stream. Elbashbeshy et al. (2010) have analyzed heat transfer over an unsteady porous stretching surface embedded in a porous medium with variable heat flux with heat source or sink. Angirasa et al. (1997) have investigated heat and mass transfer by natural convection with opposing buoyancy effects in a fluid saturated porous medium. The dissipation effects on MHD non linear flow and heat transfer past a porous surface with prescribed heat flux have been investigated by Devi and Ganga (2010). Reddy and Reddy (2011)have studied the mass transfer and heat generation effects on MHD free convection flow past an inclined vertical surface in a porous medium.

The problems of heat and mass transfer in combination with chemical reaction are of great importance in many processes and have attracted the attention of many researchers. A reaction is said to be of the order $\mathrm{n}$, if the reaction rate is proportional to the n-power of concentration. In particular, a reaction is said to be first-order, if the rate of reaction is directly proportional to concentration itself. In well mixed system, the reaction is heterogeneous if it takes place at an interface and homogeneous, if it takes place in solution. The effect of chemical reaction on free convection and heat transfer past an oscillating infinite vertical plate has been studied by Muthucumaraswamy and Meenakshisundaram (2006). Anjalidevi and Kandasamy (2000) have analyzed the effect of chemical reaction on MHD flow with heat and mass transfer past a semi-infinite plate. Choudhury and Jha (2008) have investigated the same on MHD micropolar fluid flow in slip flow regime. Al-Odat and Al-Azab (2007) have studied the influence of chemical reaction on transient MHD free convection over a moving vertical plate. Kandasamy et al. (2005) have investigated the influence of chemical reaction on MHD flow with heat and mass transfer over a vertical stretching sheet in presence of heat source and thermal stratification effect. Ahmed (2010) has analyzed the effect of chemical reaction on transient MHD free convective flow over a vertical plate in slip flow regime. Bala et al. (2012) have investigated the radiation effects on MHD flow past an exponentially accelerated isothermal vertical plate with uniform mass diffusion in the presence of heat source. Baoku et al. (2012) have analyzed the influence of thermal radiation on a transient MHD Couette flow through a porous medium. Basu et al. (2011) have studied the radiation and mass transfer effects on transient free convection flow of dissipative fluid past semi-infinite vertical plate with uniform heat and mass flux. Muthucumaraswamy and Chandrakala (2006) have analyzed the radiation, heat and mass transfer effects on moving isothermal vertical plate in presence of chemical reaction. Rao et al. (2012) have found out the chemical effects on an unsteady MHD free convection fluid past a semi-infinite vertical plate embedded in a porous medium with heat absorption. El-Aziz (2009) has investigated the radiation effect on the flow and heat transfer over an unsteady stretching sheet. Sandeep et al. (2012) have investigated the effect of radiation chemical reaction on transient MHD free convective flow over a vertical plate through porous medium. Singh et al. (2010) have studied the effect of thermal radiation and magnetic field on unsteady stretching permeable sheet in presence of free stream velocity. Suneetha et al. (2010) have analyzed the radiation and mass transfer effects on MHD free convective dissipative fluid in the presence of heat source/sink.

The applications of the mechanisms of nonNewtonian fluid flows in modern technology and industries have attracted the researchers in a large scale. Keeping in view of the applications and the important roles played by the non-Newtonian fluid flow mechanisms in various manufacturing processes, authors like Kelly et al. (1999), Subhash et al. (2001), Sonth et al.(2002), Abel et al. (2007), Choudhury and Mahanta (2009), Choudhury and Dey (2010), Choudhury and Das (2012) etc. have analyzed some problems of physical interest in this field.

The objective of the present paper is to study the free convective transient MHD flow of nonNewtonian fluid characterized by Walters liquid (Model $B^{\prime}$ ) with heat and mass transfer in presence of radiation and chemical reaction past a vertical plate in a porous medium when a transverse variable suction velocity is applied on the porous plate and to observe the visco-elastic effects on the fluid flow field along with other flow parameters involved in the problem.

The constitutive equation for Walters liquid (Model $\mathrm{B}^{\prime}$ ) is

$\sigma^{\mathrm{ik}}=-\mathrm{pg}_{\mathrm{ik}}+2 \eta_{0} \mathrm{e}^{\mathrm{ik}}-2 \mathrm{~K}_{0} \mathrm{e}^{\mathrm{ik}}$

where $\sigma^{\mathrm{ik}}$ is the stress tensor, $\mathrm{p}$ is isotropic pressure, $g_{i k}$ is the metric tensor of a fixed coordinate system $x^{i}, v^{i}$ is the velocity vector, the contravariant form of $\mathrm{e}^{\text {ik }}$ is given by

$\mathrm{e}^{\prime \mathrm{ik}}=\frac{\partial \mathrm{e}^{\mathrm{ik}}}{\partial \mathrm{t}}+\mathrm{v}^{\mathrm{m}} \mathrm{e}^{\mathrm{ik}}{ }_{\mathrm{m}}-\mathrm{v}^{\mathrm{i}}{ }_{\mathrm{m}} \mathrm{e}^{\mathrm{im}}-\mathrm{v}^{\mathrm{i}}{ }_{\mathrm{m}} \mathrm{e}^{\mathrm{mk}}$

It is the convected derivative of the deformation rate tensor $\mathrm{e}^{\mathrm{ik}}$ defined by

$2 \mathrm{e}^{\mathrm{ik}}=\mathrm{v}_{, \mathrm{k}}^{\mathrm{i}}+\mathrm{v}^{\mathrm{k}}{ }_{, \mathrm{I}}$

Here $\eta_{0}$ is the limiting viscosity at the small rate of shear which is given by $\eta_{0}=\int_{0}^{\infty} \mathrm{N}(\tau) \mathrm{d} \tau$ and $\mathrm{K}_{0}=\int_{0}^{\infty} \tau \mathrm{N}(\tau) \mathrm{d} \tau$

$\mathrm{N}(\tau)$ being the relaxation spectrum as introduced by Walters (1960.1962). This idealized model is a valid approximation of Walters liquid (Model B') taking very short memories into account so that terms involving 
$\int_{0}^{\infty} \tau^{\mathrm{n}} \mathrm{N}(\tau) \mathrm{d} \tau, \quad \mathrm{n} \geq 2$

have been neglected.

\section{MATHEMATICAL FORMULATION}

A two dimensional unsteady free convective MHD flow of Walters liquid (Model B') with heat and mass transfer past a semi-infinite heated vertical porous plate immersed in a porous medium in presence of chemical reaction and radiation has been considered. The $\bar{x}$-axis is considered vertically upwards along the plate and $\bar{y}$-axis is taken normal to it. Let $\bar{u}$ and $\bar{v}$ be the fluid velocity along $\bar{x}$ and $\bar{y}$ directions respectively. A homogeneous chemical reaction of first order of constant rate $\bar{K}_{2}$ is assumed to exist between the diffusing species and the fluid. A transverse uniform magnetic field is applied normal to the plate.

We restrict our investigation to the following conditions:

i) the induced magnetic field can be neglected as the transverse applied magnetic field and the magnetic Reynolds number are very small for metallic liquids and partially ionized fluids,

ii) no electric field is present as there is no applied voltage,

iii) Soret and Dufour effects are negligible as the concentration of diffusing species is very small in comparison with other chemical species,

iv) temperature of the fluid is governed by energy equation involving radiative heat flux and species concentration is governed by concentration equation involving chemical reaction of first order.

With the above assumptions and the Boussinesq's approximation, the equations governing the fluid flow and heat and mass transfer are as follows:

equation of continuity:

$\frac{\partial \overline{\mathrm{v}}}{\partial \overline{\mathrm{y}}}=0$

Equation (6) shows that the suction velocity at the plate is either a constant or a function of time only. So, assuming the suction velocity to be oscillatory about a non-zero constant mean, one can write $\bar{v}=-v_{0}\left(1+\varepsilon A e^{i \bar{\omega} \bar{t}}\right)$

Where $v_{0}$ is the mean suction velocity and $\varepsilon, \mathrm{A}$ are such that $\varepsilon \mathrm{A} \ll 1$. The negative sign indicates that the suction velocity is directed towards the plate.

momentum equation:

$\frac{\partial \bar{u}}{\partial \bar{t}}-\bar{v} \frac{\partial \bar{u}}{\partial \bar{y}}=v \frac{\partial^{2} \bar{u}}{\partial \bar{y}^{2}}-\frac{K_{0}}{\rho}\left\{\frac{\partial^{3} \bar{u}}{\partial \bar{t} \partial \bar{y}^{2}}-\bar{v} \frac{\partial^{3} \bar{u}}{\partial \bar{y}^{3}}\right\}+$
$g \beta\left(\bar{T}-\bar{T}_{\infty}\right)+g \bar{\beta}_{c}\left(\bar{C}-\bar{C}_{\infty}\right)-\frac{v \bar{u}}{\bar{K}}-\frac{\sigma B_{0}{ }^{2} \bar{u}}{\rho}$

Or,

$\frac{\partial \bar{u}}{\partial \bar{t}}-v_{0}\left(1+\varepsilon A e^{i \bar{\omega} \bar{t}}\right) \frac{\partial \bar{u}}{\partial \bar{y}}=v \frac{\partial^{2} \bar{u}}{\partial \bar{y}^{2}}-\frac{K_{0}}{\rho}\left\{\frac{\partial^{3} \bar{u}}{\partial \bar{t} \partial \bar{y}^{2}}-\right.$

$\left.v_{0}\left(1+\varepsilon A e^{i \bar{\omega} \bar{t}}\right) \frac{\partial^{3} \bar{u}}{\partial \bar{y}^{3}}\right\}+g \beta\left(\bar{T}-\bar{T}_{\infty}\right)+$ $g \bar{\beta}_{c}\left(\bar{C}-\bar{C}_{\infty}\right)-\frac{v \bar{u}}{\bar{K}}-\frac{\sigma B_{0}{ }^{2} \bar{u}}{\rho}$

(8) where $v=\frac{\eta_{0}}{\rho}$.

energy equation:

$\frac{\partial \bar{T}}{\partial \bar{t}}-v_{0}\left(1+\varepsilon A e^{i \bar{\omega} \bar{t}}\right) \frac{\partial \bar{T}}{\partial \bar{y}}=-\frac{\kappa}{\rho C_{p}} \frac{\partial^{2} \bar{T}}{\partial \bar{y}^{2}}-\frac{\partial \bar{q}_{r}}{\partial \bar{y}}$

concentration equation:

$\left.\frac{\partial \bar{C}}{\partial \bar{t}}-v_{0}\left(1+\varepsilon A e^{i \bar{\omega} \bar{t}}\right) \frac{\partial \bar{C}}{\partial \bar{y}}=D \frac{\partial^{2} \bar{C}}{\partial \bar{y}^{2}}-\bar{K}_{2}\left(\bar{C}-\bar{C}_{\infty}\right) 10\right)$

The boundary conditions are

$\bar{y}=0: \bar{u}=\bar{L} \frac{\partial \bar{u}}{\partial \bar{y}}, \bar{T}=\bar{T}_{w}+\varepsilon\left(\bar{T}_{w}-\bar{T}_{\infty}\right) e^{i \bar{\omega} \bar{t}}$,

$\bar{C}=\bar{C}_{w}+\varepsilon\left(\bar{C}_{w}-\bar{C}_{\infty}\right) e^{i \bar{\omega} \bar{t}}$

$\bar{y} \rightarrow \infty: \bar{u} \rightarrow 0, \bar{T} \rightarrow \bar{T}_{\infty}, \bar{C} \rightarrow \overline{\mathrm{C}}_{\infty}$

Using Rosseland approximation, which is valid for optically thick fluids, we get that $\bar{q}_{r}=-\frac{4 \sigma_{s}}{3 k_{e}} \frac{\partial \bar{T}^{4}}{\partial \bar{y}} \quad$ where $\sigma_{s}$ is the Stefan Boltzmann constant and $k_{e}$ is the mean absorption coefficient.

Expanding $\bar{T}^{4}$ into the Taylor series about $\bar{T}_{\infty}$ which after neglecting higher order terms takes the form $\bar{T}^{4} \cong 4 \bar{T}_{\infty}^{3} \bar{T}-3 \bar{T}_{\infty}^{4}$

On introducing the non-dimensional quantities

$y=\frac{v_{0} \bar{y}}{v}, u=\frac{\bar{u}}{v_{0}}, t=\frac{\bar{t} v_{0}{ }^{2}}{4 v}, \omega=\frac{4 \bar{\omega} v}{v_{0}{ }^{2}}$,

$P_{r}=\frac{\mu C_{p}}{\kappa}, \theta=\frac{\bar{T}-\bar{T}_{\infty}}{\bar{T}_{w}-\bar{T}_{\infty}}, \phi=\frac{\bar{C}-\bar{C}_{\infty}}{\bar{C}_{w}-\bar{C}_{\infty}}$,

$G_{r}=\frac{v g \beta\left(\bar{T}_{w}-\bar{T}_{\infty}\right)}{v_{0}{ }^{3}}, G_{m}=\frac{v g \bar{\beta}_{c}\left(\bar{C}_{w}-\bar{C}_{\infty}\right)}{v_{0}{ }^{3}}, M=\frac{\sigma B_{0}{ }^{2} v}{\rho v_{0}{ }^{2}}$,

$S_{c}=\frac{v}{D}, h=\frac{v_{0} \bar{L}}{v}, K_{r}=\frac{v \bar{K}_{2}}{v_{0}{ }^{2}}, K=\frac{\bar{K} v_{0}^{2}}{v^{2}}$,

$R=\frac{\kappa k_{e}}{4 \sigma_{s} \bar{T}_{\infty}{ }^{3}}$

Equations (8) to (10) and the boundary conditions (11) become

$\frac{1}{4} \frac{\partial u}{\partial t}-\left(1+\varepsilon A e^{i \omega t}\right) \frac{\partial u}{\partial y}=\frac{\partial^{2} u}{\partial y^{2}}-K_{1}\left\{\frac{1}{4} \frac{\partial^{3} u}{\partial t \partial y^{2}}-\right.$
$\left.\left(1+\varepsilon A e^{i \omega t}\right) \frac{\partial^{3} u}{\partial y^{3}}\right\}+G_{r} \theta+G_{m} \phi-M u-\frac{u}{K}$

where $K_{1}=\frac{K_{0} v_{0}^{2}}{\rho v^{2}} \cdot \frac{1}{4}$.

$\frac{\partial \theta}{\partial t}-\left(1+\varepsilon A e^{i \omega t}\right) \frac{\partial \theta}{\partial y}=\frac{1}{P_{r}}\left(1+\frac{4}{3 R}\right) \frac{\partial^{2} \theta}{\partial y^{2}}$

$\frac{1}{4} \frac{\partial \phi}{\partial t}-\left(1+\varepsilon A e^{i \omega t}\right) \frac{\partial \phi}{\partial y}=\frac{1}{S_{c}} \frac{\partial^{2} \phi}{\partial y^{2}}-K_{r} \phi$

The modified boundary conditions are

$\mathrm{y}=0: u=h \frac{\partial u}{\partial y}, \theta=1+\varepsilon e^{i \omega t}, \phi=1+\varepsilon e^{i \omega t}$
$y \rightarrow \infty: u \rightarrow 0, \theta \rightarrow 0, \phi \rightarrow 0$

For $\varepsilon \ll 1$, introducing the perturbation scheme $f(y, t)=f_{0}(y)+\varepsilon e^{i \omega t} f_{1}(y)+o\left(\varepsilon^{2}\right)$

where $f$ represents $u, \theta$ and $\phi$ in Eqs. (14) to (16) and comparing the coefficients of various powers of $\varepsilon$ and neglecting those of second and higher powers of $\varepsilon$ we get the following equations.

\subsection{Zeroth-Order Equations}


R. Choudhury and S. Kumar Das. / JAFM, Vol. 7, No. 4, pp. 603-609, 2014.

$$
\begin{aligned}
& K_{1} \frac{d^{3} u_{0}}{d y^{3}}+\frac{d^{2} u_{0}}{d y^{2}}+\frac{d u_{0}}{d y}-\left(M+\frac{1}{K}\right) u_{0}=-G_{r} \theta_{0}- \\
& G_{m} \phi_{0} \\
& \frac{d^{2} \theta_{0}}{d y^{2}}+N P_{r} \frac{d \theta_{0}}{d y}=0 \\
& \frac{d^{2} \phi_{0}}{d y^{2}}+S_{c} \frac{d \phi_{0}}{d y}-K_{r} S_{c} \phi_{0}=0 \\
& \text { where }=\frac{1}{1+\frac{4}{3 R}}
\end{aligned}
$$

\subsection{First-Order Equations}

$K_{1} \frac{d^{3} u_{1}}{d y^{3}}+\left(1-\frac{i \omega K_{1}}{4}\right) \frac{d^{2} u_{1}}{d y^{2}}+\frac{d u_{1}}{d y}-\left(M+\frac{1}{K}+\frac{i \omega}{4}\right) u_{1}$

$=-G_{r} \theta_{1}-G_{m} \phi_{1}-A \frac{d u_{0}}{d y}-K_{1} A \frac{d^{3} u_{0}}{d y^{3}}$

$\frac{d^{2} \theta_{1}}{d y^{2}}+N P_{r} \frac{d \theta_{1}}{d y}-\frac{i \omega P_{r} N}{4} \theta_{1}=-A N P_{r} \frac{d \theta_{0}}{d y}$

$\frac{d^{2} \phi_{1}}{d y^{2}}+S_{c} \frac{d \phi_{1}}{d y}-S_{c}\left(\frac{i \omega}{4}+K_{r}\right) \phi_{1}=-A \frac{d \phi_{0}}{d y}$

The corresponding boundary conditions are

$y=0: u_{0}=h \frac{d u_{0}}{d y}, u_{1}=h \frac{d u_{1}}{d y}, \theta_{0}=1, \theta_{1}=1$,

$\phi_{0}=1, \phi_{1}=1$

$y \rightarrow \infty: u_{0} \rightarrow 0, u_{1} \rightarrow 0, \theta_{0} \rightarrow 0, \theta_{1} \rightarrow 0$,

$\phi_{0} \rightarrow 0, \phi_{1} \rightarrow 0$

To solve Eqs. (19) and (22) we apply another perturbation scheme for $K_{1} \ll 1$ as

$u_{0}=u_{00}+K_{1} u_{01}+o\left(K_{1}^{2}\right)$

$u_{1}=u_{10}+K_{1} u_{11}+o\left(K_{1}^{2}\right)$

and we get the following equations.

Zeroth- order equations:

$$
\begin{aligned}
& \frac{d^{2} u_{00}}{d y^{2}}+\frac{d u_{00}}{d y}-\left(M+\frac{1}{K}\right) u_{00}=-G_{r} \theta_{0}-G_{m} \phi_{0} \\
& \frac{d^{2} u_{10}}{d y^{2}}+\frac{d u_{10}}{d y}-\left(M+\frac{1}{K}+\frac{i \omega}{4}\right) u_{10} \\
& =-G_{r} \theta_{1}-G_{m} \phi_{1}-A \frac{d u_{00}}{d y}
\end{aligned}
$$

First-order equations:

$$
\begin{aligned}
& \frac{d^{2} u_{01}}{d y^{2}}+\frac{d u_{01}}{d y}-\left(M+\frac{1}{K}\right) u_{01}=-\frac{d^{3} u_{00}}{d y^{3}} \\
& \frac{d^{2} u_{11}}{d y^{2}}+\frac{d u_{11}}{d y}-\left(M+\frac{1}{K}+\frac{i \omega}{4}\right) u_{11}=-\frac{d^{3} u_{10}}{d y^{3}}+ \\
& \frac{i \omega}{4} \frac{d^{2} u_{10}}{d y^{2}}-A \frac{d u_{01}}{d y}-A \frac{d^{3} u_{00}}{d y^{3}}
\end{aligned}
$$

The corresponding boundary conditions are $y=0: u_{00}=h \frac{d u_{00}}{d y}, u_{01}=h \frac{d u_{01}}{d y}, u_{10}=\frac{d u_{10}}{d y}$, $u_{11}=h \frac{d u_{11}}{d y}$

$y \rightarrow \infty: u_{00} \rightarrow 0, u_{01} \rightarrow 0, u_{10} \rightarrow 0, u_{11} \rightarrow 0$

Solutions of Eqs. (20), (21), (23), (24) and (27) to (30) are

$$
\begin{aligned}
& \theta_{0}=e^{-N P_{r} y} \\
& \phi_{0}=e^{-q_{2} y} \\
& (33) \\
& \theta_{1}=b_{1} e^{-q_{1} y}+\left(1-b_{1}\right) e^{-N P_{r} y} \\
& \phi_{1}=b_{2} e^{-q_{3} y}+b_{3} e^{-q_{2} y} \\
& u_{00}=b_{4} e^{-q_{4} y}+b_{5} e^{-N P_{r} y}+b_{6} e^{-q_{2} y} \\
& u_{01}=b_{7} e^{-q_{4} y}+b_{8} y e^{-q_{4} y}+b_{9} e^{-N P_{r} y}+ \\
& b_{10} e^{-q_{2} y}
\end{aligned}
$$

$$
\begin{aligned}
& u_{10}=b_{16} e^{-q_{5} y}+b_{11} e^{-q_{1} y}+b_{12} e^{-N P_{r} y}+ \\
& b_{13} e^{-q_{2} y}+b_{14} e^{-q_{3} y}+b_{15} e^{-q_{4} y} \\
& u_{11}=b_{17} e^{-q_{5} y}+b_{18} y e^{-q_{5} y}+b_{19} e^{-q_{1} y}+ \\
& b_{20} e^{-N P_{r} y}+b_{21} e^{-q_{2} y}+b_{22} e^{-q_{3} y} \\
& \quad+b_{23} e^{-q_{4} y}+b_{24} y e^{-q_{4} y} \\
& \text { Equations }(18) \text { and (26) yield } \\
& \theta=\theta_{0}+\epsilon e^{i \omega t} \theta_{1} \\
& \phi=\phi_{0}+\epsilon e^{i \omega t} \phi_{1} \\
& u=u_{00}+K_{1} u_{01}+\epsilon e^{i \omega t}\left(u_{10}+K_{1} u_{11}\right)
\end{aligned}
$$

The non-dimensional skin-friction coefficient $\sigma_{0}$ at the plate $\mathrm{y}=0$ is given by

$$
\begin{aligned}
& \sigma_{0}=u_{00}{ }^{\prime}(0)+K_{1} u_{01}{ }^{\prime}(0)+\epsilon e^{i \omega t}\left\{u_{10}{ }^{\prime}(0)+\right. \\
& \left.K_{1} u_{11}{ }^{\prime}(0)\right\}-\frac{i K_{1} \epsilon \omega e^{i \omega t}}{4}\left\{u_{10}{ }^{\prime}(0)+K_{1} u_{11}{ }^{\prime}(0)\right\}+ \\
& K_{1}\left(1+\epsilon A e^{i \omega t}\right)\left[u_{00}{ }^{\prime(0)}+K_{1} u_{01}^{\prime(0)}+\right. \\
& \left.\epsilon e^{i \omega t}\left\{u_{10}{ }^{\prime \prime}(0)+K_{1} u_{11}{ }^{\prime \prime}(0)\right\}\right]
\end{aligned}
$$

The non-dimensional form of the rate of heat transfer in the form of Nusselt number $\mathrm{N}_{\mathrm{u}}$ is given by,

$$
N_{u}=\left(\frac{\partial T}{\partial y}\right)_{y=0}=\left(T_{0}{ }^{\prime}+\varepsilon e^{i \omega t} T_{1}{ }^{\prime}\right)_{y=0}
$$

The non-dimensional form of the rate of mass transfer at the plate in terms of Sherwood number $S_{h}$ is given by

$$
S_{h}=\left(\frac{\partial C}{\partial y}\right)_{y=0}=\left(C_{0}{ }^{\prime}+\varepsilon e^{i \omega t} C_{1}\right)_{y=0}
$$

The constants are obtained but not given here due to brevity.

\section{DISCUSSION OF THE RESULTS}

The purpose of the present study is to find out the visco-elastic effects on transient MHD flow with heat and mass transfer in presence of radiation and chemical reaction of Walters liquid (Model B') past a vertical plate in a porous medium. The viscoelastic effect is characterized by the non zero values of the non dimensional parameter $\mathrm{K}_{1}$ whereas $\mathrm{K}_{1}=0$ represents the Newtonian fluid flow phenomenon.

All the numerical calculations are to be carried out for $\mathrm{P}_{\mathrm{r}}=3, \mathrm{G}_{\mathrm{r}}=5, \mathrm{G}_{\mathrm{m}}=5, \mathrm{~h}=.1, \mathrm{~K}=.1, \varepsilon=.001, \omega=1$, $\omega \mathrm{t}=\pi / 2$ throughout the problem.

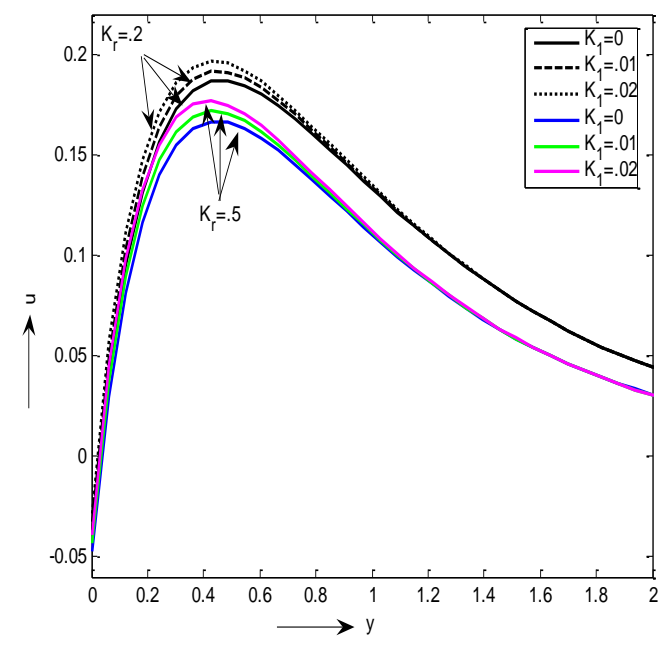

Fig.1. Fluid velocity $u$ against $y$ for $R=.2, M=1$, $\mathrm{A}=.5, \mathrm{~S}_{\mathrm{c}}=.1$ 
R. Choudhury and S. Kumar Das. / JAFM, Vol. 7, No. 4, pp. 603-609, 2014.

Figure 1 illustrates that the fluid velocity $u$ enhances with the growth of the visco-elastic parameter $\mathrm{K}_{1}$ and diminishes with the rise of chemical reaction parameter $\mathrm{K}_{\mathrm{r}}$. It has also been noticed that the magnitude of the velocity profile against y first increases up to a certain value and then begins to diminish till it becomes zero at large distance from the plate $\mathrm{y}=0$ for both non-Newtonian and Newtonian fluid flows .

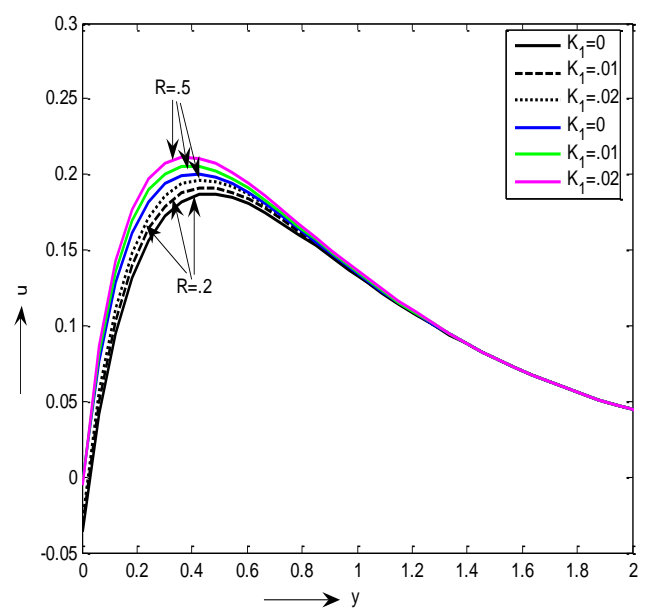

Fig. 2. Fluid velocity $u$ against $y$ for $K_{r}=.2, M=1$, $\mathrm{A}=.5, \mathrm{~S}_{\mathrm{c}}=.1$

Figure 2 describes that the fluid velocity $u$ has an accelerating trend with the rising effect of the viscoelastic parameter $K_{1}$ and the radiation parameter $R$ as well. It is also observed that the magnitude of the fluid velocity against y first increases up to a certain value and then begins to diminish till it becomes zero at large distance from the plate $\mathrm{y}=0$ for both non-Newtonian and Newtonian fluid flows. .

Figures 3, 4 and 5 illustrate the behavior of skinfriction coefficient $\sigma_{0}$ at the plate $\mathrm{y}=0$ against the suction parameter $\mathrm{A}$, the magnetic parameter $\mathrm{M}$ and the Schmidt number $S_{c}$ respectively.

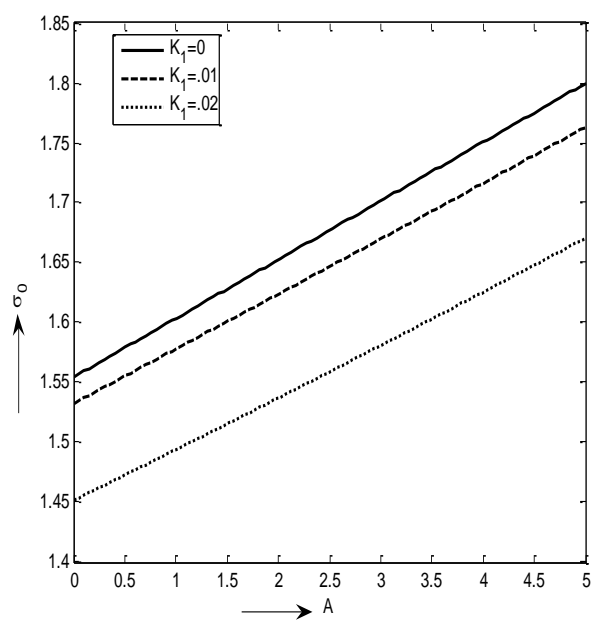

Fig.3. Skin friction coefficient $\sigma_{0}$ against $A$ on the plate $y=0$ for $R=.2, K_{r}=.2, M=1, S_{c}=.1$
Figure. 3 illustrates that the skin friction coefficient $\sigma_{0}$ at the plate $\mathrm{y}=0$ against the suction parameter $\mathrm{A}$

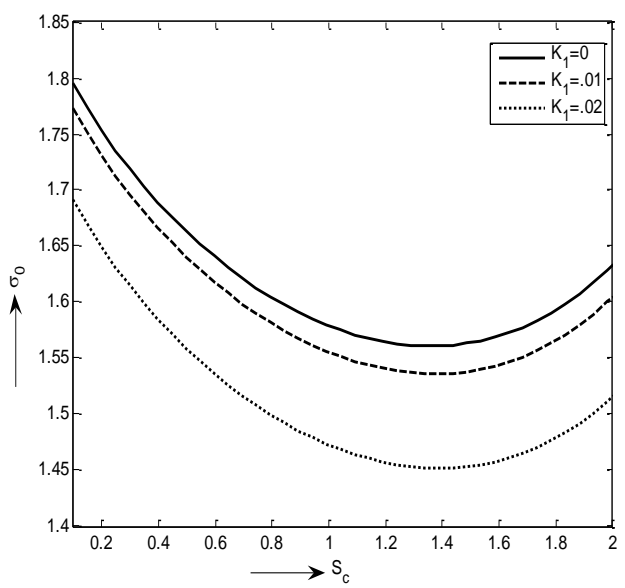

diminishes with the growing effect of the viscoelastic parameter $K_{1}$ but enhances with the increase of A.

Figure. 4 depicts that the skin friction coefficient $\sigma_{0}$ at the plate $\mathrm{y}=0$ against the magnetic parameter $\mathrm{M}$ diminishes with the rising effect of both the viscoelastic parameter $K_{1}$ and the magnetic parameter $M$.

Fig.4. Skin friction coefficient $\sigma_{0}$ against $M$ on the plate $\mathrm{y}=0$ for $\mathrm{R}=.2, \mathrm{~K}_{\mathrm{r}}=.2, \mathrm{~A}=.5, \mathrm{~S}_{\mathrm{c}}=.1$

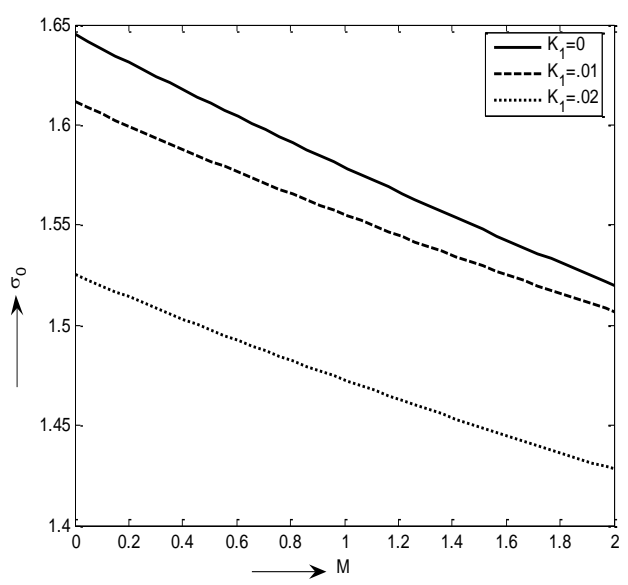

Fig.5. Skin friction coefficient $\sigma_{0}$ against $S_{c}$ on the plate $\mathrm{y}=0$ for $\mathrm{R}=.2, \mathrm{~K}_{\mathrm{r}}=.2, \mathrm{~A}=.5, \mathrm{M}=1$

Figure. 5 exhibits that the skin friction coefficient $\sigma_{0}$ at the plate $\mathrm{y}=0$ against the Schmidt number $\mathrm{S}_{\mathrm{c}}$ diminishes with the growth of the visco-elastic parameter $K_{1}$. It is also found that the magnitude of the skin-friction coefficient at the plate $\mathrm{y}=0$ against the Schmidt number $S_{c}$ first decreases up to a certain value of $S_{c}$ and then increases in the interval $(.1,2)$.

\section{CONCLUSION}

An analysis of free convective transient flow of a visco-elastic fluid characterized by Walters liquid (Model B') over a vertical porous plate through porous media in presence of radiation and chemical 
reaction with heat and mass transfer is presented when a transverse uniform magnetic field normal to the plate and a transverse variable suction velocity are applied on the porous plate for different values of visco-elastic parameter $\mathrm{K}_{1}$ in combination of other flow parameters.

This study leads to the following conclusions:

* The velocity field is considerably affected by the visco-elastic parameter along with other flow parameters.

* The velocity field has an accelerating trend with the growing effect of the visco- elastic parameter.

* The velocity field exhibits an increasing trend with the enhancement of the radiation parameter.

* The velocity field diminishes with the rise of the chemical reaction parameter.

* The skin friction coefficient shows a decelerating trend with the rise of the viscoelastic parameter against all of suction parameter, magnetic parameter and Schmidt number.

* decelerating trend with the rise of the viscoelastic parameter against all of suction parameter, magnetic parameter and Schmidt number.

\section{REFERENCES}

Abel, M. S., P. G. Siddheshwar, and M. M. Nandeeppanavar (2007). Heat transfer in a visco-elastic boundary layer flow over a stretching sheet with viscous dissipation and non-uniform heat source. Int. J. Heat and Mass Transfer. 50(5-6), 960-966.

Angirasa, D., G. P. Peterson, and I. Pop (1997). combined heat and mass transfer by natural convection with opposing buoyancy effects in a fluid saturated porous medium. Int. J. Heat Mass Trans. 40(12), 2755-773.

Ahmed, S. (2010). Influence of chemical reaction on transient MHD free Convective flow over a vertical plate in slip-flow Regime. Emirates Journal for Engineering Research. 15(1), 2534.

Anjalidevi, S. P. and R. Kandasamy (2000). Effects of a chemical reaction heat and mass transfer on MHD flow past a semi infinite plate. Z. Angew. Math. Mech. 80, 697-701.

Al-Odat, M. Q. and Al-Azab (2007). Influence of chemical reaction on transient MHD free convection over a moving vertical plate. Emirates J. Engg. Res. 12(3), 15-21.

Bala, P., A. Reddy, N. B. Reddy, and S. Suneetha (2012). Radiation effects on MHD flow past an exponentially accelerated isothermal vertical plate with uniform mass diffusion in the presence of heat source. JAFM, 5(3), 119-126.

Baoku, I. G., C. I. Cookey, and B. I. Olajuwon (2012). Influence of thermal radiation on a transient MHD Couette flow through a porous medium. JAFM, 5(1), 81-87.

Basu, B., V. R. Prasad, and N. B. Reddy (2011). Radiation and mass transfer effects on transient free convection flow of dissipative fluid past semi-infinite vertical plate with uniform heat and mass flux. JAFM, 4(1), 1526.

Chaudhary, R. C. and A. K. Jha (2008). Effects of chemical reactions on MHD micropolar fluid flow past a vertical plate in slip-flow regime. Applied Mathematics and Mechanics. 29(9), 1179-1194.

Choudhury, R. and D. Dey (2010). Free convective visco-elastic flow with heat and mass transfer through a porous medium with periodic permeability. Int. J. Heat and Mass Transfer (Elsevier). 53, 1666-1672.

Choudhury, R. and M. Mahanta. (2009). Free convective MHD flow of a visco- elastic fluid past an infinite vertical channel. Int. J. Appl. Math. 23, 189-203.

Choudhury, R. and U. Das (2012). Heat transfer to MHD oscillatory visco-elastic flow in a channel filled with porous medium. Physics Research International. 2012,Ar ID 8795537, 5 pages.

Devi, S. P. A., and B. Ganga (2010).Dissipation effects on MHD non linier flow and heat transfer past a porous surface with prescribed heat flux. JAFM, 3(1), 1-6.

El-Aziz, M. A. (2009). Radiation effect on the flow and heat transfer over an unsteady stretching sheet. International Communications in Heat and Mass Transfer. 36(5), 521- 524.

Elbashbeshy, E. M. A., D. M. Yassmin, and A. A. Dalia (2010). Heat transfer over an unsteady porous stretching surface embedded in a porous medium with variable heat flux in the presence of heat source or sink".African Journal of Mathematics and Computer Science Research.3(5), 68-73.

Kandasamy, R., K. Periasamy, and K. K. S. Prabhu (2005). Chemical reaction, heat and mass transfer on MHD flow over a vertical stretching surface with heat source and thermal stratification effects. Int. J. Heat and Mass Transfer. 48, 21-22, 4557-4561.

Kelly, D., K. Vajravelu, and L. Andrews 1999. Analysis of heat mass transfer of a viscoelastic, electrically conducting fluid past a continuous stretching sheet. Non-linear 
R. Choudhury and S. Kumar Das. / JAFM, Vol. 7, No. 4, pp. 603-609, 2014.

Analysis: Theory, Methods and Applications. 36(6), 767-784.

Muthucumaraswamy, R. and P. Chandrakala, (2006). Radiation heat and mass transfer effects on moving isothermal vertical plate in the presence of chemical reaction. J. of Appl.Mech. and Engng. 11(3), 639-646.

Muthucumaraswamy, R. and S. Meenakshisundaram (2006). Theoretical study of chemical reaction effects on vertical oscillating plate with variable temperature. Theoret. Appl. Mech. 33(3), 245-257.

Rao, J. A., S. Sivaiah, and R. S. Raju (2012). Chemical effects on an unsteady MHD free convection fluid past a semi-infinite vertical plate embedded in a porous medium with heat absorption. JAFM, 5(3), 63-70.

Reddy, M. G. and N. B. Reddy (2011). Mass transfer and heat generation effects on MHD free convection flow past an inclined vertical surface in a porous medium. $J A F M, 4(2), 7-11$.

Sandeep, N., A. V. B. Reddy, and V. Sugunamma (2012). Effect of Radiation and Chemical Reaction on Transient MHD Free Convective Flow over a Vertical Plate Through Porous Media. Chem. and Process Engng Res. 2, 1-9.

Sharma, P. K. (2005). Fluctuating thermal and mass diffusion on unsteady free convective flow past a vertical plate in slip-flow regime. Latin American Applied Research. 35, 313319.

Singh, P., N. S. Tomer, and D. Sinha (2010a). Numerical study of heat transfer over stretching surface in porous media with transverse magnetic field. Proceeding of International Conference on Challenges and application of Mathematics in Sciences and Technology. 422-430.

Singh, P., N. S. Tomer, S. Kumar, and D. Sinha (2010b). MHD oblique stagnation-point flow towards a stretching sheet with heat transfer. International Journal of Applied Mathematics and Mechanics. 6(13), 94-111.

Singh, P., A. Jangid, N. S. Tomer, S. Kumar, andD. Sinha (2010c). Effects of Thermal Radiation and Magnetic Field on Unsteady Stretching Permeable Sheet in Presence of Free Stream Velocity.Int. J.of Information and Mathematical Sci. 6(3), 63-69.

Sonth, R. M., S. K. Khan, M. S. Abel, and K. V. Prasad (2002). Heat and mass transfer in a visco-elastic flow over an accelerating surface with heat source/sink and viscous dissipation. Heat and Mass Transfer. 38(3), 213-220.

Subhash, A. M., A. Joshi, and R. M. Sonth (2001). Heat transfer in MHD visco-elastic fluid flow over a stretching surface. Zeitschrift für angewandte Mathematikund Mechanik. 81, 10, 691-698.

Suneetha, S., N. B. Reddy, and V. R. Prasad (2010). Radiation and mass transfer effects on MHD free convective dissipative fluid in the presence of heat source/sink. JAFM, 4(1), 107113.

Walters, K. (1960). The motion of an elasticoviscous liquid contained between co-axial cylinders (II). Quart. J. Mech. Appl. Math. $13,444-461$.

Walters, K. (1962). The solution of flow problems in the case of materials with memories. $J$. Mecanique. $\quad 1, \quad 473-478$. 